NOUVEAUTE :

\title{
les relations
}

franco-allemandes

I 8 I 5-I975

\author{
RAYMOND POIDEVIN \\ professeur à l'Université de Metz \\ JACQUES BARIÉTY \\ professeur à l'Université de Strasbourg
}

De Mme de Staël au général de Gaulle, de Metternich à Briand, de Bismarck à Poincaré, de Marx à Renan, de Foch à Hitler, l'histoire des relations entre la France et l'Allemagne ne manque pas de figures de premier plan. En I6o ans, quatre guerres, trois occupations de la France et deux occupations de l'Allemagne ont mis aux prises les deux nations. Histoire d'affrontements et d'agressions, de peurs, voire de hantises, mais aussi de mirages, d'espoirs et de nostalgies, les relations franco-allemandes de I8I5 à I 975 concernent les vécus conscients et inconscients des deux peuples; elles constituent aussi l'un des axes, et parfois le principal, des relations internationales.

Deux historiens français, l'un et l'autre spécialistes de l'Allemagne et des relations franco-allemandes, jettent un regard ferme et serein, sinon détaché, sur cette histoire tumultueuse et souvent passionnelle d'antagonismes et d'admirations réciproques.

Collection $U$, série "Histoire contemporaine " dirigée par René Rémond

\section{armand colin}




\title{
REVUE ÉCONOMIQUE
}

\author{
Publication bimestrielle \\ avec le concours de l'École des Hautes Études en Sciences Sociales \\ et du Centre National de la Recherche Scientifique
}

Vol. XXVIII

No 3 - Mai 1977

Y. Simon, H. Tezenas du Montcel, De la théorie à la réforme de l'entreprise

C. Montmarquette, Ramdom walk Behavior of Finished Goods Inventory Investment : Some Theoretical and Empirical Considerations

R. M. GELPI, La formation des taux et le mécanisme de la distribution de crédits dans le système bancaire français avant et après la réforme de 1965-1967

J. Fontanel, Conceptualisation de la simulation dans l'analyse macroéconomique

J.-P. Simonin, Une nouvelle approche de la technique du survivant

Vol. XXVIII

No 4 - Juillet 1977

P. Bauchet, Les mystères de la méso-économie : l'exemple du transport maritime

J. Debenest, Le temps de consommation - variable macroéconomique

Paul Vidonne, Une erreur de P. Sraffa : La rente et l'étalon avec ressource rare homogène

J.-P. LAFFaRGUE, La spéculation sur le marché des changes : définition et paradoxes

J. GADREY, La linéarité de la frontière du prix des facteurs

J.-P. Simonin, Le surplus de productivité globale et la norme d'efficacité intersectorielle

J. de BANDT, Surplus de productivité globale et norme d'efficacité intersectorielle quelques éléments de réflexion et de réponse

Abonnements 1977 : France 160 F. - Étranger 180 F. - Le numéro $30 \mathrm{~F}$.

Étudiants et assistants, France seulement : $100 \mathrm{~F}$ (joindre à la commande une photocopie de la carte d'étudiant ou de la carte professionnelle d'assistant en cours de validité et au nom de l'abonné.)

Librairle Armand COLIN, 103, boulevard Saint-Michel, 75240 Paris Cedex 5.

C.C.P. Paris $21335-25$ 
NOUVEAUTÉ:

\title{
LA GAULE ROMAINE \\ Lucien Lerat \\ U2 $n^{\circ} 234: \quad F$
}

Dans la même collection :

\author{
SOCIÉTÉ ET ÉCONOMIE \\ DE LA REPUBLIQUE ROMAINE \\ Louis Harmand \\ U2 $n^{\circ} 232: 24,80 \mathrm{~F}$ \\ LES INSTITUTIONS ROMAINES \\ de la Rome royale à la Rome chrétienne \\ Jean Rougé \\ U2 $n^{\circ} 73: 25,90 \mathrm{~F}$ \\ LE DROIT PRIVÉ ROMAIN \\ Jean Gaudemet \\ U2 n० $220: 30,50 \mathrm{~F}$

\section{LA RELIGION ROMAINE} \\ Marcel Le Glay \\ U2 $n^{\circ} 168: 24,80 \mathrm{~F}$

\section{LE PREMIER SIËCLE DE NOTRE ĖRE} \\ Paul Petit \\ U2 no $47: 21,70 \mathrm{~F}$

\section{LE BAS EMPIRE} \\ André Chastagnol \\ U2 no $58: 21,70 \mathrm{~F}$
}

Chacun de ces ouvrages est composé de deux parties : une introduction d'ensemble et un important choix de textes, traduits des auteurs anciens, annotés et présentés suivant les mêmes divisions que la première partie. 


\section{THE \\ ECONOMIC HISTORY \\ REVIEW}

Second Series, Volume XXX, No. 2, May 1977

CONTENTS

\section{Articles}

Douglass C. North and Robert Paul Thomas, The First Economic Revolution

A. R. Disney, The First Portuguese India Company, I628-33

Roger Anstey, The Slave Trade of the Continental Powers, I760-18ro

Ian Donnachie, Sources of Capital and Capitalization in the Scottish Brewing Industry, c. $175^{\circ}-1830$

CORMAC ó GRÁdA, The Beginnings of the Irish Creamery System, I880-I9I4

Elizabeth Roberts, Working-Class Standards of Living in Barrow and Lancaster, I890-I9I4

W. R. Garside, Juvenile Unemployment and Public Policy between the Wars Comment

Eric Kerridge, The Coal Industry in Tudor and Stuart England: A Comment

D. C. Coleman, The Coal Industry: A Rejoinder

Essays in bibliography and criticism

LXXVIII. The Model Game D. C. Coleman

Reviews

Published for The Economic History Society by

The Broadwater Press Ltd, Welwyn Garden City, Hertfordshire, England 
Centre de Recherches Historiques

Civilisations et Sociétés 55

\author{
G U Y T H U I L L I E R
}

\title{
POUR UNE HISTOIRE DU QUOTIDIEN AU XIXe SIELCLE EN NIVERNAIS
}

\author{
Préface de Paul Leuilliot
}

\begin{abstract}
L'auteur cherche à établir les bases d'une histoire du vécu quotidien, en partant d'exemples nivernais : la pollution de l'air et de l'eau, l'hygiène corporelle, l'accouchement, le médicament, les gestes, les travaux ménagers, la lessive, la perception du temps, les bruits, les couleurs, les matériaux, le réveil, les sígnaux, le tablier : autant d'évolutions retracées minutieusement de I80o à I970. Ce livre touche à la fois à l'histoire des mentalités, à l'histoire sociale et économique, à l'histoire du corps, à l'histoire démographique, à l'histoire des techniques, à l'histoire administrative.

Des notes de méthodes indiquant les sources et les difficultés de la recherche forment en annexe un véritable guide des sources de l'histoire du quotidien.
\end{abstract}

1977. $\mathrm{xxIV}+492$ p. $165 \mathrm{~F}$.

$\mathrm{MOUTON}$

Difusion en France : LIBRAIRIE DE LA NOUVELle FacUlté 3O RUE DES SAINTS PËRES - 75007 PARIS

PUBLICATIONS ORIENTALISTES DE FRANCE POF - Études

FRANCINE HERAIL

FONCTIONS ET FONCTIONNAIRES JAPONAIS AU DÉBUT DU Xle SIËCLE

Pour la première fois en français, un ouvrage capital de référence sur les institutions gouvernementales et administratives du Japon de l'an mil. Une minutieuse analyse des textes définissant et régissant les structures de l'État est doublée de l'examen de documents divers. II s'en dégage une vision concrète, et souvent très différente du prétendu modèle chinois, d'un pouvoir de nature cérémonielle plutôt que politique.

Cet ouvrage doit intéresser quiconque travaille sur les structures politiques et institutionnelles du Moyen Age, qu'il soit juriste, historien ou comparatiste.

2 volumes $14,5 \times 21.938$ pages dont 144 pages d'index. $120 \mathrm{~F}$.

En vente en librairie, ou envoi franco de port sur commande accompagnée du règlement (125 $\mathrm{F}$ pour un envoi recommandé).

Publications Orientalistes de France -2 rue de Lille - 75007 Paris

CCP : ALC - La Source 3381655 C 


\title{
Revue française de Sociologie
}

\author{
Vol. XVII, No 2, Avril-Juin 1976
}

\section{A propos de DURKHEIM}

\section{TEXTES INÉDITS OU INCONNUS D'ÉMILE DURKHEIM réunis par Philippe BESNARD}

- Lettres à Célestin Bouglé

- Rôle des Universités dans l'éducation sociale du pays

- Autres textes inconnus

Mohamed Cherkaour, Socialisation et conflit : les systèmes éducatifs et leur histoire selon Durkheim

Bernard LACROIX, La vocation originelle d'Émile Durkheim

Pierre Birnbaum, La conception durkheimienne de l'État : l'apolitisme des fonctionnaires

Jean-Claude Filloux, "Il ne faut pas oublier que je suis fils de rabbin "

Victor Karady, Durkheim, les sciences sociales et l'Université : bilan d'un semi-échec

Philippe Besnard, Anti- ou anté-durkheimisme? Contribution au débat sur les statistiques officielles du suicide

\section{BIBLIOGRAPHIE DURKHEIMIENNE}

Bibliographie des écrits sur Durkheim, par Philippe Besnard et Marie-France ESSYAD

Comptes rendus

ADMINISTRATION, ABONNEMENTS : ÉDITIONS DU C.N.R.S.

15, quai Anatole-France, 75700 Paris - Tél. : 555-92-25

L'abonnement part du 1 er janvier de chaque année

Tarif : L'abonnement ........... $90 \mathrm{~F}$

Le numéro $\ldots \ldots \ldots \ldots \ldots \quad 28 \mathrm{~F}$

C.C.P. Paris 9131-53, Éditions du Centre national de la Recherche scientilique 


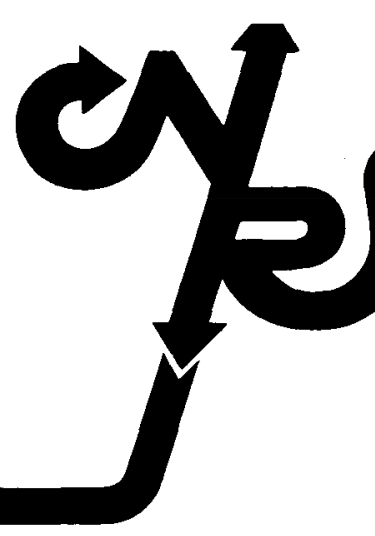

CENTRE DE DOCUMENTATION SCIENCES HUMAINES / DU $5^{\mathrm{e}}$ SIECLE A I95 8

\title{
BIBLIOGRAPHIE ANNUELLE DE L'HISTOIRE DE FRANCE
}

\author{
(T. XXI/I975)
}

C. ALBERT-SAMUEL, B. MOREAU, S. POSTEL

Recensement en ro I9I notices des textes parus en I975; plus de I 700 périodiques, les actes de I23 congrès, IOI volumes de Mélanges.

- manuels généraux et sciences auxiliaires de l'histoire; histoire politique de la France; histoire des institutions; histoire économique et sociale; histoire religieuse ; la France d'Outre-mer ; histoire de la civilisation ; histoire locale

- périodiques dépouillés (59 pages)

- index matières (I6o pages)

- table des noms d'auteurs (7I pages)

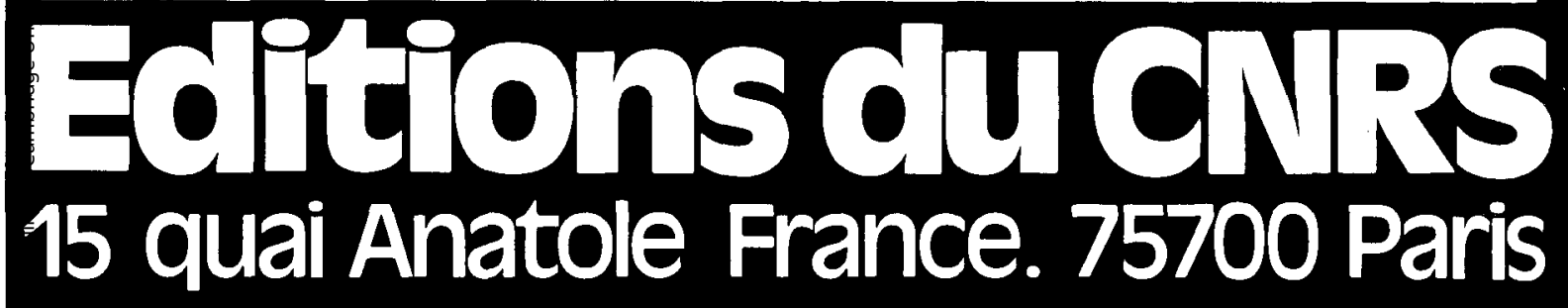

CCP. Paris 9061-11 - Tél. 555-92-25

$\mathrm{M}$

profession

adresse

achète le livre chez son libraire

à défaut aux Editions du CNRS (chèque joint)

et demande votre documentation

$\square$ Sciences humaines

Sciences exactes et naturelles

Trésor de la langue Française

Revue de l'Art 


\section{RE V UE \\ TIERS - MO N DE}

Tome XVIII, $n^{\circ} 70$

Avril-Juin 1977

James H. MITTELMAN, De la dépendance et des relations entre l'armée et le pouvoir civil

Serge LATOUCHE, Transferts de plus-value et échange inégal

Michel GODET et Jacques ARCADE, Critique des termes de l'échange et conséquences pour le Tiers-Monde

David L. McKEE, Róle des facteurs extérieurs sur l'infrastructure des pays en voie de développement

Groupe de recherche de l'IEDES, Commerce extérieur et organisation de l'espace : un essai de typologie des espaces dominés

André GRJEBINE, La spécialisation internationale : coûts probables pour les pays industrialisés

Philippe BERNARD, Pour une généralisation de l'étude des facteurs sociaux et culturels du développement

Panayotis ROUMELIOTIS, La politique des prix d'importation et d'exportation des entreprises multinationales en Grèce

Bruno BEKOLO ELIE, Une analyse de 15 ans d'échanges entre pays africains 1960-1975

Michel BOURGEOIS, Attitudes et comportements de la population rurale sénégalaise vis-à-vis des moyens audio-visuels de formation

Michel CARTILLIER, La révolution verte en Inde et le rôle des petites industries: le cas des pompes d'irrigation

François LABOUESSE, Point de vue sur l'évolution de l'agriculture soviétique depuis 1929

\section{CHRONIQUE INTERNATIONALE}

Nhü Lê ABADIE, Nouvelle stratégie de lutte contre la pauvreté à la Conférence mondiale de l'emploi. - Genève, 4-17 juin 1976

Michel LELART, L'Assemblée générale du Fonds monétaire international. - Manille, 4-8 octobre 1976

\section{BIBLIOGRAPHIE}

Direction-Rédaction : Instltut d'Étude du Développement Économique et Social, 58, boulevard Arago - 75013 PARIS - Tél. : 331-28-01

Abonnements et vente : Presses Universitalres de France, 12, rue Jean-de-Beauvais 75005 PARIS - Tél. : 033-48-03 - C.C.P. : PARIS n 1302-69

France et Communauté : $110 \mathrm{~F}$. Étranger : $128 \mathrm{~F}$. 


\section{TERRE HUMAINE \\ collection dirigée par JEAN MALAURIE \\ Quelques titres consacrés aux Amérindiens}

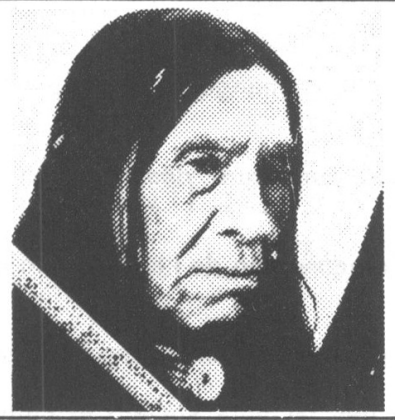

\section{Tahca Ushte \\ Richard Erdoes}

\section{DE MEMOIRE INDIENNE}

\section{La vie d'un Sioux voyant et guérisseur}

Dénonçant les tares de l'aveugle civilisation technicienne, Tahca Ushte, dans sa quête du savoir, dans son message destiné à chacun d'entre nous, n'écrit pas seulement un livre du passé. II nous donne des Mémoires d'avenir, un livre de vie et de sagesse puisé aux sources résurgentes de la tradition amérindienne.

\section{Claude Lévi-Strauss / TRISTES TROPIQUES}

Un livre humain, un grand livre. Peu d'ouvrages soulèvent des problèmes aussi vastes, aussi fondamentaux.

Don C. Talayesva / SOLEIL HOPI

Je ne vois guère de grands personnages dont les Mémoires soient préférables à ceux de ce modeste héros.

\section{Theodora Kroeber / ISHI}

ANDRE PIEYRE DE MANDIARGUES (N.R.F.)

"Ishi" est sans doute le livre le plus bouleversant qu'ii m'ait été donné de lire. Seul "le journal d'Anne Frank", peut-être...

YVES BERGER (LE MONDE)

\section{Richard Lancaster / PIEGAN}

Livre remarquable et témoignage exceptionnel sur le triste crépuscule des Amérindiens.

\section{Jacques Soustelle / LES QUATRE SOLEILS}

En des pages éblouissantes, les plus importantes de toute son œuvre, Jacques Soustelle nous offre sa propre vision du monde.

ANDRE BRISSAUD (LE PARISIEN LIBERE)

\section{Francis Huxley / AIMABLES SAUVAgeS}

Recueil d'anecdotes, somme de légendes, précis d'ethnologie appliquée, "Aimables sauvages", comme tous les livres qui traitent d'un groupe en voie de disparition, est un témoignage désolé.

\section{Ettore Biocca / YANOAMA}

YVES BERGER (L'EXPRESS)

L'un des livres les plus exceptionnels publiés au cours des dernières décennies. Un ouvrage incomparale, comme est incomparable l'expérience qu'il restitue.

GEORGES BALANDIER (LA QUINZAINE LITTERAIRE)

\section{Pierre Clastres / CHRONIQUE DES INDIENS GUAYAKI}

Mangeurs d'hommes, ils l'étaient passionnément... La "Chronique" se lit comme le chant funèbre des derniers Guayaki.

\section{Jean Malaurie / LES DERNIERS ROIS DE THULE}

Les Esquimaux du Groenland racontés par un savant et un poète. La maîtrise de l'écrivain, l'art de la mise en scène conduisent à une réflexion sur les hommes qui relèvent le défi de la géographie.

$$
\text { MAX GALLO (L'EXPRESS) }
$$

\section{Plon}




\section{QUADERNI STORICI}

ANNO XII FASCICOLO I GENNAIO-APRILE 1977

\section{Letteratura ideologia società negll Anni Trenta \\ A cura di Franco Marenco}

F. M., PREMESSA

Cesare CASES, L'autocritica degli intellettuali tedeschi e il dibattito sull'espressionismo Claudio MAGRIs, La "prima realtà " di Doderer

Vittorio STRADA, Unione Sovietica : dalla letteratura proletaria al realismo socialista

Elementi di dibattito: A. Caracciolo, Un'ipotesi di periodizzazione ; L. Secci, Totalitarismo nello stato e nella famiglia

Carmelo Samona', Gli intellettuali spagnoli della seconda repubblica e la cultura dell' "impegno"

Dario Puccini, America Latina : il prezzo dell'autocoscienza

Elementi di dibattito: A. Melis, Spunti di ricerca sull'America Latina; M. Isnenghi, Trenta-Quaranta : l'ipotesi della continuità

Alberto Asor Rosa, L' "impegno " nella letteratura italiana tra fascismo e postfascismo Jacqueline Risset, André Breton : un surrealismo socialista?

Elementi di dibattito: U. Piscopo, Un filone scomodo, il surrealismo in Italia; R. Runcini, Cinema e letteratura nella Francia del Fronte popolare

Franco Marenco, La tradizione come ideologia in tre periodici inglesi : Criterion, Scrutiny, The left review

Vito Amoruso, Stati Uniti : dialettica di un'integrazione

Elementi di dibattito: G. Sertoli, L'ideologia nella letteratura; S. Sabbadini, Ideologia $\mathrm{e}$ intellettuali o ideologia della produzione intellettuale?; $\mathrm{G}$. Pagliano Ungari, Editoria, forma letteraria, impegno ; B. Placido, Il romanzo keynesiano

\section{DISCUSSIONI E DIBATTITI}

Raffaele RomaneldI, Storia politica e storia sociale dell'Italia contemporanea : problemi aperti

Pasquale Villani, Problemi e prospettive di ricerca : la storia sociale dell'Italia contemporanea

Guido SAVAREse, Note sulla " ricostruzione " in Italia

Lucio GAMBI, Le " regioni " italiane come problema storico

Libri ricevuti ; English Summaries

QUADERNI STORICI, Via A. Musa, 6-ooI6I Roma

La sede redazionale è presso l'Istituto di studi storici e sociologici della

Facoltà di Economia e Commercio, Palazzo degli Anziani, 6oroo Ancona.

Amministrazione ed edizione : Società editrice IL MULINO,

Via Santo Stefano, 6 - 40125 Bologna. 


\section{autrement}

Direction-RAdection

\section{- NOUVEAUX ROLES INDIVIDUELS, NOUVELLES PRATIQUES DE GROUPE}

- Quand je fais mon autocritique

- Premiers pas en équipe, premiers obstacles

- Itineraire d'une innovation

- Ces médecins qui ne jouent pas au docteur...

- Tours, Bruxeliles : "maisons" et

"boutiques" de santé

- Au coeur des conflits, la Maison Médicale de Grenoble

- Au Québec, une révolution?

- Nous, les infirmières

- S.M.G., M.A.S., ... et les enfants de 68

\section{- DU NOUVEAU DANS LES INSTITUTIONS DE PREVENTION}

- Echapper aux pièges de la médecine scolaire

- P.M.I., sous-medecine ou avant-garde ?

- La grande peur des médecins du travail

- Une altermative, l'action du G.I.S.

- Les pionniers des centres paralieles pour adolescents

- Une prévention"démédicalisée" ?

Quel becu rêve !

\section{- REINVENTER LA FORMATION}

- Une approche inhabituelle de l'enseignement

- Des experiences innovatrices : Bobigny, Maastricht

\section{- LES "APPAREILS" ET LES ACTEURS}

- Crise des institutions et pouvoir medical

- A droite, à gauche,

quel discours, quel projet?

- La révolution verbale des mandarins

- Autres diagnostica, autres ordonnances

- Pour le public : toubib, professeur ou guérisseur?

- Aujourd'hui encore, les secrets des grands-meres

\section{- REGARDS}

- Quand la médicalisation devint populaire

- Complexes et fantames du sociologue médical

- La muture Ilitich

- Quatre vêrités sur la santé
Michel Leyers

Jean-Yves Chetaille

Pierre Guillet

Gerard Mauger, Claude Fosaé

Des collectifo

Gerard Mouger, Cloude Fosse

François Canonne

Marie-Frangoise colliere

Jacques Goudard, ALain Lebas Jacqueline de Chambrun

Suzanne Kepes

Marion Duval

Christian Nils Robert

Alain Letourmy

Jean-Jacques Guilbert

Hélene Ploix

Pierre Comillot,P. Pissarro

\section{Frangois Steudler}

Michel Legros, France Lert Serge Karsenty

N. Bensald, J. Benveniste,

J. Caissel, J.CZ. Henrard,

W. Junod, L. Lareng,

H. Pradal, J.Ch. Sournia

Jean-Pierre Corbeau

Francoibe Loux

Jacques Léonard

Claudine Hersich

J. Bouberot, A. Collomp,

J.P. Dupuy, B. Ginisty,

D. Monchicourt, J. Revel

Pierre Cornillot 


\section{EUROPEAN JOURNAL OF SOCIOLOGY}

Numéros spéciaux

SPECIAL ISSUES

Sondernümmern

I I Industrial Society and Representative Government.

2 A la recherche des classes perdues.

II I Le sabre et la loi.

2 On the Welfare State.

III I-2 Universität im Umbau: Anpassung oder Widerstand?

IV I In Quest of Political Participation.

2 Trois études sur la science.

V I Organisation der Macht, Macht der Organisation.

2 Tocqueville, Marx, Weber.

VI I Simulation in Sociology.

2 Armed Forces and Society in Western Europe.

VII I Aliénation et structure or Conscience and Consciousness. On Suicide.

VIII I Paradoxes of Transitional Societies.

2 Sympathy for Alien Concepts.

IX I Weber et Durkheim: le solitaire et le chef d'école.

2 Zur Problematik der Modernisierung or The Concept of Modernity.

X I Survivances et permanences or Continuity and Re-enactment.

2 Myths and Mass Media.

XI I La planification dans des nations du tiers monde : bilan critique.

2 La foi et les mœurs or Faith and Morals.

XII I Permanent non-Revolution.

2 Reflections on Durkheim.

XIII I Permanent non-Revolution (continued).

2 "A Sack of Potatoes"?

XIV I The Political Elite, British and French.

2 Tantum Religio...

XV I Faith and Power.

2 Citoyens armés, prétoriens désarmés.

XVI I Observer's Analysis of Caste and Clientele.

2 From Madness to Mental Illness.

XVII I Structure and Interest.

2 Professions avec ou sans compétence.

$$
\text { RÉDACTION }
$$

Raymond Aron - Jean Baechler

Ralf Dahrendorf - Éric de Dampierre

Ernest Gellner - Steven Lukes

Musée de l'Homme, 75II6 Paris

Address for subscriptions: Cambrigde University Press, London

Annual Subscription : $£ 7 \cdot 50$

Number of the year : $€ 4 \cdot 00$

Back Number $\quad: £ 7.50$ 


\title{
RAISON PRÉSENTE
}

\author{
Directeur : Victor LEDUC
}

Revue trimestrielle $-\mathrm{No}_{4}^{2 / 1977}$

Les nouveaux Gouroux en question : Alain UTUJI, L'irrationalisme en philosophie aujourd'hui. - Alain LAGARDE, Nietzsche et le rationalisme

Dr. Jacques WAYNBERG, Visages de la sexologie

Charles Maignial, Divination de Jésus ou dédivinisation de Dieu ?

Laura LÉvi MACARIUS, Magie et sacrifice humain

Daniel BACHET, la délinquance juvénile

Le numéro : $16 \mathrm{~F}$.

\section{Éditions Rationalistes}

r6, rue de l'École Polytechnique - 75005 PARIS

\section{The Shelby Cullom Davis Center for Historical Studies}

\author{
Princeton University. - Director: Lawrence Stone
}

\section{THE HISTORY OF THE PROFESSIONS}

For two years, 1978-79 and 1979-80, the subject of the Davis Center Seminar will be The History of the Professions. The Seminar will stress the broad and changing relationship of professionalization to society through a series of studies of particular professions. Focussing mainly on the history of Western civilization over the past thousant years, the Seminar hopes to deal with the central problems of change over time such as:

how and why have the professions developed; what have been their growth periods, and for what reasons; and what have been the results of growth upon society, politics, administration and systems of value. The answers to these questions will raise others concerning how a profession is defined; how it is organized, with what prescribed norms and sanctions; and what are the areas of conflict, both within it and between it and outside institutions, such as the University, the Church and the State.

The Center will offer a limited number of Research Fellowships for one or two semesters, running from September to January and from February to June, designed for highly recommended younger scholars, as well as for senior scholars with established reputations. Candidates who have not both finished their dissertations and had at least a year of full-time teaching experience thereafter are not eligible. It will also offer one Teaching Fellowship for the whole year, funded by N.E.H., the recipient of which will teach an undergraduate seminar for one term at Princeton, as well as carrying on research and attending the Seminar. Fellows are expected to live in Princeton in order to take an active part in the intellectual interchange with other members of the Seminar. Funds are very limited, and candidates are, therefore, strongly urged to apply to other grantgiving institutions as well as the Center.

Inquiries and requests for Fellowship Application Forms should be addressed to the Secretary, Davis Center for Historical Studies, I 29 Dickinson Hall, Princeton University, Princeton, New Jersey, 08540, U.S.A. The deadline for fellowship applications and for letters of recommendation for $1978-79$ is December, I, 1977. Scholars who are not applicants for Fellowships but would like to visit Princeton to offer a paper to the Seminar are asked to write to the Director. 


\section{études rurales}

Revue trimestrielle

Vient de paraître un nouveau numéro spécial :

\section{Pouvoir et Patrimoine au Village}

(Première partie : 63-64 juillet-décembre 1976)

(Deuxième partie : 65 janvier-mars I977)

Un colloque interdisciplinaire sur ce thème a été organisé à Strasbourg, en octobre I975, par l'Association des ruralistes français, qui inaugurait ainsi son activité scientifique. Ces deux fascicules d'un numéro spécial ont été préparés à partir des communications présentées. Les intitulés des cinq sections du sommaire résument les facettes principales d'un phénomène complexe que l'actualité politique et administrative française vient de placer au premier plan : les mécanismes du pouvoir dans l'univers villageois :

Première partie : L'institution municipale rurale. - Histoire de la politique et des pouvoirs au village.

Deuxième partie : Les mécanismes de la transmission des patrimoines - Société globale, État et vie communale rurale. - Le nouveau pouvoir technique (encadrement, animation).

Les abonnements (4 numéros) peuvent être souscrits auprès de votre libraire ou auprès de la Librairie de la Nouvelle Faculté, 30, rue des Saints-Pères, 75007 Paris

Institutions et libraires : $120 \mathrm{~F}$.

Vente au numéro: $30 \mathrm{~F}$.

Particuliers : 8o F.

\section{MOUTON}




\section{PRESSES UNIVERSITAIRES DE GRENOBLE}

Domaine Universitaire - B.P. 47 X - 38040 Grenoble-Cédex - Tél. (76) 42.18 .72

\section{COLLECTION HISTOIRE}

- MEMOIRES DUUN MILITANT OUVRIER DU CREUSOT 1841-1905

de J.B. Dumay, établi et commenté par G. Ponsot

432 p., Br., 16 × 24, Coédition François Maspéro - PUG 1976

Pierre Ponsot présente et annote avec rigueur et précision ce témoignage d'un fils du peuple qui parle de sa vie, une vie difficile de travail et de luttes, d'un ouvrier de b grande industrie qui fait revivre le monde du capitalisme industriel triomphant en cette seconde moitié du XIXe siècle, à travers le Creusot, Schneider : une usine, une ville, une famille. Et c'est aussi un témoignage d'un militant républicain, socialiste qui, en retraçant ses luttes et ses actions quotidiennes, évoque l'histoire du mouvement ouvrier et des grandes étapes de la lutte des classes au $19 \mathrm{e}$ siècle.

\section{ACTUALITES}

- L'UNIVERSITE OUVERTE : Les dossiers de Vincennes présentés par Debeauvais

299 p., Br., $16 \times 24$

Vincennes : une universitê différente des autres, aussi célèbre à l'étranger qu'en France mais avec les images les plus contradictoires : Expérience pédagogique de pointe, ou Université rouge? Université ouverte, ou ghetto expérimental ? Université de travailleurs, ou asile des anciens combattants de Mai 68 ?

Université critique du monde contemporain, ou entreprise de bradage des diplómes a des non-bacheliers ? Modèle de travail individualisé par petits groupes, ou foyer d'agitation et de grèves permanentes? Université modèle ou Univer sité poubelle ?

La presse parle souvent de Vincennes, mais presque toujours dans les moments de crise. Au-delà du sensationnel, quelle est la vérité de la vie quotidienne ? Après sept années d'existence, toujours précaire, Vincennes survit et reste différente des autres universités. Il était temps de faire un bilan de l'expérience.

Pour ce faire, Vincennes a ouvert tous ses dossiers, sans auto-censure. Des rapports, des bilans, des tracts, des débats d'enseignants et d'êtudiants, des enquêtes, un ensemble de textes plus vrais qu'un plaidoyer.

- LA PRESSE GRENOBLOISE DE LA LIBERATION, 1944-1952

de $B$. Montergnole

256 p., Br., $16 \times 24,1974$

L'Histoire de la presse dans le dauphiné au lendermain de la libération, c'est I'histoire d'une espérance déçue, celle de l'existence de plusieurs journaux comme le "Travailleur Alpin", "Les Allobroges" puis "Le Réveil", peu à peu remise en cause par les déchirures des mouvements et partis unis pendant la Résistance et par les impératifs économiques. Et c'est l'histoire d'un journal "Le Dauphiné Liberé" qui prend son essor et qui symbolise la presse à monopole le système du journal unique et souvent de pensée.. unique

\section{DIFFUSION :}

Paris : OPhrYs, 10, rue de Nesle, Paris 6e - Tol. 326.82 .04

Pour la Suisse : L'AGE D'HOMME, 10, Métropole, 1003 Lausanne

Pour le Bónélux : NOUVELLE DIFFUSION, 8 rue du Chătelain, 1050 Bruxelles

Pour l'Allemagne et l'Autriche : DOKUMENTE VERLAG, Postfach 1340, D 7600 Offenburg 


\section{En lan mille la France avait déjà 2000 ans d'histoire...}

\section{LES ORIGINES DE LA FRANCE}

par Ferdinand Lot, de l'Institut.

\section{Histoire de la Gaule et de la naissance de la France}

Oeuvre maîtresse d'un très grand historien qui consacra sa vie à l'étude de notre plus lointain passé, LES ORIGINES DE LA FRANCE est un livre capital pour l'histoire de la formation de la France : c'est, en effet, le seul ouvrage historique actuel qui offre un tableau d'ensemble de la Gaule indépendante et romaine, en même temps qu'une histoire complète de la naissance de la France, depuis la conquête des Francs, le règne des Mérovingiens et des Carolingiens jusqu'à l'avènement des Capétiens en 987.

PLAN DE L'OUVRAGE Tome 1

La Gaule celtique

et la conquête romaine

De l'an 1000 avant notre ère au IIle siécle après $\mathbf{J}$. C. - Les 60 peuplcs - La brillante société gallo-romain Tome 2 La Gaule romaine et les invasions germaniques $(290$ à 491 après J C.) L'assimilation romaine - Les premiers martyrs chrétiens Pancs, Alamans, Alains, Burgondes, Wisigoths

$$
\text { Tome } 3
$$

Naissance de la France : les Merovingiens
$(480$ à 740 apres J.C.)

Clovis et les Francs saliens - Pendant 3 siècles 36 rois se disputent l'Etat palais - Charles Martel.

$$
\begin{gathered}
\text { Tome } 4 \\
\text { Charlemagne et les Carolingiens }
\end{gathered}
$$
$(740-1000)$

Les 46 ans du long et prestigieux regne de Charlemagne - L'invasion Tome 5

La civilisation carolingienne et l'An mille

Les capitulaires - Les missi dominici Economique - Les écoles - Les premiers féodaux.

EDITION EXCLUSIVE EN SOUSCRIPTION

Aux Editions Tallandier :

17, rue Remy-Dumoncel- 75680 Paris Cedex 1
En 5 volumes, Ferdinand Lot raconte 20 siècles d'une aventure fabuleuse et inconnue commencée 1000 ans avant l'ère chrétienne, quand la France s'appelait la Gaule :

Vercingetorix, premier patriote de notre histoire, Saint-Martin, Sainte-Genevieve, Sainte-Clotilde dont le pouvoir sur le coeur d'un homme allait avoir de si grandes conséquences pour l'avenir de la France. Clovis, baptisé à Reims avec ses 3000 guerriers, Dagobert et ses conseillers Saint-Eloi et Saint-Ouen, la victoire Dagobert et historique de Charles Martel a Poitiers, Charlemagne, l'empe-
reur légeñdaire et son règne glorieux...

CE vous fera découvrir leurs vrais visages, leurs vraies vies et leurs oeuvres.

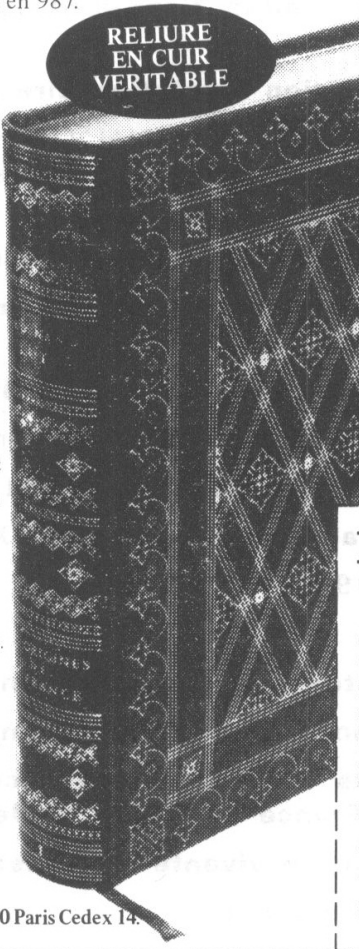

BON D’EXAMEN GRATUIT

\section{à renvoyer aux EDITIONS TALLANDIER}

17, rue Remy-Dumoncel - 75680 PARIS CEDEX 14

Oui, je désire recevoir pour un Si je désire souscrire, je garderai le examen gratuit de 10 jours le pre- premier volume et vous règlerai mier tome des ORIGINES DE LA son prix : $86,00 \mathrm{~F}(+4,90 \mathrm{~F}$ de frais FRANCE par Ferdinand Lot dans d'envoi). Je recevrai à mesure les sa premiere édition de luxe reliee 4 tomes suivants, a raison d un par plein cuir et illustree. Cet examen mois et je les règlerai après chaque cst sans conditions, et je peux vous reception au meme prix que le retourner ce premier volume dan NOM

\section{ADRESSE}

SIG̣NATURE (indispensable) 21 


\section{CAHIERS DES ANNALES}

En vente à la LIBRAIRIE ARMAND COLIN, 103, boulevard Saint-Michel, 75005 PARIS Compte chèques postaux: Paris, No $21335-25$

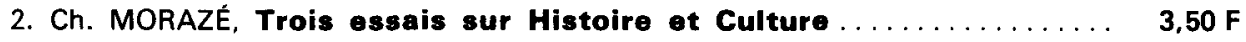

6. L. E. HALKIN, Initiation a Ia critique historique, $4^{\mathbf{\theta}}$ édition revue et corrigée

7. G. DEBIEN, En haut Poitou : defricheurs au travail (XV•-XVI! siecles) $5,50 \mathrm{~F}$

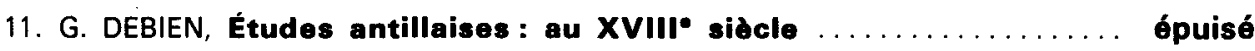

13. M. BLOCH, La France sous les derniers Capétiens (1223-1328)

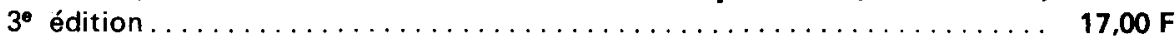

14. M. DOMMANGET, BIanqui et I'opposition révolutionnaire a la fin du Second Empire $\ldots \ldots \ldots \ldots \ldots \ldots \ldots \ldots \ldots \ldots \ldots \ldots \ldots \ldots$

15. J. BARNAVE, Introduction a la Révolution française (présentation de

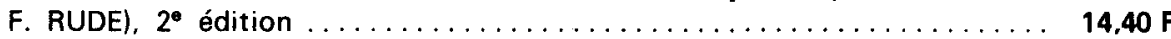

16. M. BLOCH, Seigneurie française et manoir anglais, $2^{\circ}$ édition $\ldots \ldots 16,20 \mathrm{~F}$

17. M. DELAFOSSE et C. LAVEAU, Le commerce du sel de Brouage aux XVII et XVII" siecles

13,40 F

20. H. ANTONIADIS-BIBICOU, Recherches sur les douanes a Byzance... $35,10 \mathrm{~F}$

22. T. PLATTER, Autobiographie (texte traduit et présenté par Marie HELMER) 16,20 F

24. M. MORINEAU, Jauges et méthodes de jauge anciennes et modernes $18,30 \mathrm{~F}$

25. J.-P. ARON, Essai sur la sensibilité alimentaire a Parie au XIX• siecle $28,80 \mathrm{~F}$

26. M. BLOCH, Souvenirs de guerre, $1914-1915 \ldots \ldots \ldots \ldots, 00 \mathrm{~F}$

27. Archéologie du village déserte, 2 volumes $\ldots \ldots \ldots \ldots \ldots \ldots \ldots, 5$ F

28. J.-J. HÉMARDINQUER, Pour une histoire de l'alimentation ........ $56.10 \mathrm{~F}$

29. G. et M. VOVELLE, Vision de la mort et de l'au-dela en Provence $26,10 \mathrm{~F}$

30. M. MORINEAU, Les faux-semblants d'un démarrage 6conomique : agriculture et démographie en France au XVII• siecle ....... $63,90 \mathrm{~F}$

31. H.-D. MANN, Lucien Febvre. La pensée vivante d'un historien ... 43,10 F

32. J. MEUVRET, Études d'histoire Economique . . . . . . . . . . . 50,80 F

34. M. MALOWIST, Croissance et régression en Europe (XIV•-XVI!* siècles)

$58,70 \mathrm{~F}$

35. W. FRIJHOFF et D. JULIA, École et sociés dans la France d'Ancien Régime 


\section{ÉCOLE DES HAUTES ÉTUdES EN SCIENCES SOCIALES \\ Centre de Recherches Historiques \\ CAHIERS DES ANNALES, 36}

\section{MAURICE AGULHON}

\section{LE CERCLE DANS \\ LA FRANCE BOURGEOISE |8|0-1848}

ÉTUDE D'UNE MUTATION DE SOCIABILITÉ

Le cercle est pour la France ce qu'est le club dans l'univers britannique; l'époque étudiée ici est celle où il apparaît puis se répand dans notre pays. Avant d'être adoptée, cette innovation a un peu inquiété, parce qu'elle parut d'abord liée au genre de vie et à la conception du monde de la bourgeoisie libérale. De plus, le cercle (en principe société d'agrément) était la plus pratique des formes licites de la vie d'association, ce qui fit qu'on l'utilisa parfois à d'autres fins. Envisagé sous cet angle, ce chapitre d'histoire de la vie quotidienne ouvre quelques horizons sur l'histoire de la culture et même sur celle de la politique dans le demi-siècle qui forme transition entre la chute de l'Ancien Régime et l'avènement de la démocratie moderne.

1977. 108 p. $38 \mathrm{~F}$. 